\title{
Existence of nontrivial weak solutions for $p$-biharmonic Kirchhoff-type equations
}

\author{
Jung-Hyun Bae ${ }^{1}$, Jae-Myoung Kim², Jongrak Lee ${ }^{3 *}$ and Kisoeb Park ${ }^{4}$
}

\section{"Correspondence:}

jrlee0124@ewha.ac.kr

${ }^{3}$ Institute of Mathematical Sciences,

Ewha Womans University, Seoul,

Republic of Korea

Full list of author information is

available at the end of the article

\begin{abstract}
We are concerned with the following $p$-biharmonic equations:

$$
\Delta_{p}^{2} u+M\left(\int_{\mathbb{R}^{N}} \Phi_{0}(x, \nabla u) d x\right) \operatorname{div}(\varphi(x, \nabla u))+V(x)|u|^{p-2} u=\lambda f(x, u) \text { in } \mathbb{R}^{N},
$$

where $2<2 p<N, \Delta_{p}^{2} u=\Delta\left(|\Delta u|^{p-2} \Delta u\right)$, the function $\varphi(x, v)$ is of type $|v|^{p-2} v$, $\varphi(x, v)=\frac{d}{d v} \Phi_{0}(x, v)$, the potential function $V: \mathbb{R}^{N} \rightarrow(0, \infty)$ is continuous, and $f: \mathbb{R}^{N} \times \mathbb{R} \rightarrow \mathbb{R}$ satisfies the Carathéodory condition. We study the existence of weak solutions for the problem above via mountain pass and fountain theorems.
\end{abstract}

MSC: 35J60; 35J92; 58E05

Keywords: $p$-biharmonic; Kirchhoff type; Variational method

\section{Introduction}

We are concerned with the following $p$-biharmonic equations:

$$
\Delta_{p}^{2} u+M\left(\int_{\mathbb{R}^{N}} \Phi_{0}(x, \nabla u) d x\right) \operatorname{div}(\varphi(x, \nabla u))+V(x)|u|^{p-2} u=\lambda f(x, u) \quad \text { in } \mathbb{R}^{N},
$$

where $2<2 p<N, 1<p<p_{*}:=\frac{N p}{N-2 p}, \Delta_{p}^{2} u=\Delta\left(|\Delta u|^{p-2} \Delta u\right)$ is a $p$-biharmonic operator, the function $\varphi(x, v)$ is of type $|v|^{p-2} v, \varphi(x, v)=\frac{d}{d v} \Phi_{0}(x, v)$, the potential function $V: \mathbb{R}^{N} \rightarrow$ $(0, \infty)$ is continuous, and $f: \mathbb{R}^{N} \times \mathbb{R} \rightarrow \mathbb{R}$ satisfies the Carathéodory condition.

The fourth-order differential equations arise in the study of deflections of elastic beams on nonlinear elastic foundations. Thus, they become very significant in engineering and physics. Many authors considered this type of equation in recent years, and we refer to $[9,13,27]$ and the references therein. For this reason, the existence of solutions of $p$ biharmonic equations has been studied by several authors; see $[6,8,12,15,21,24,30$, $31,34]$. To obtain the existence and multiplicity results for the $p$-Laplace type operators, which generalize the usual $p$-Laplacian, the authors in $[10,28]$ considered the following condition:

$$
d|v|^{p} \leq \varphi(x, v) \cdot v \leq p \Phi_{0}(x, v)
$$

(c) The Author(s) 2019. This article is distributed under the terms of the Creative Commons Attribution 4.0 International License (http://creativecommons.org/licenses/by/4.0/), which permits unrestricted use, distribution, and reproduction in any medium, provided you give appropriate credit to the original author(s) and the source, provide a link to the Creative Commons license, and indicate if changes were made. 
for all $x \in \mathbb{R}^{N}$ and $v \in \mathbb{R}^{N}$, and for some positive constant $d$; see also [14]. On the other hand, Kirchhoff in [20] initially proposed the following equation:

$$
\rho \frac{\partial^{2} u}{\partial t^{2}}-\left(\frac{\rho_{0}}{h}+\frac{E}{2 L} \int_{0}^{L}\left|\frac{\partial u}{\partial x}\right| d x\right) \frac{\partial^{2} u}{\partial x^{2}}=0
$$

which is a generalization of the classical D'Alembert's wave equation. Also, Woinowsky and Krieger [33] in the 1950s considered a stationary analogue of the evolution equation of Kirchhoff type, namely

$$
u_{t t}+\Delta^{2} u-M\left(\|\nabla u\|^{2}\right) \Delta u=f(x, u)
$$

as a model for the deflection of an extensible beam on nonlinear foundations. Here, $u$ denotes the displacement, $f$ is the force that the foundations exert on the beam, and $M$ models the effects of the small changes in the length of the beam (see, e.g., [3-5, 7] for the physics viewpoint model). In view of mathematics, many researchers have extensively studied the existence of weak solutions for the elliptic problem of Kirchhoff type in recent years (see, e.g., $[11,16,18])$. Based on these references, we consider the generalized elliptic equation (P) involving the $p$-biharmonic and generalized $p$-Laplacian of Kirchhoff type.

Since the seminal paper of Ambrosetti and Rabinowitz in [2], the existence of solutions for the elliptic problem has been studied by many researchers. A common feature of these works is that the following condition, which is originally due to Ambrosetti and Rabinowitz, is imposed on the nonlinearity $f$ :

(AR) There exist positive constants $m$ and $\zeta$ such that $\zeta>p$ and

$$
0<\zeta F(x, t) \leq f(x, t) t \quad \text { for } x \in \Omega \text { and }|t| \geq m \text {, }
$$

where $F(x, t)=\int_{0}^{t} f(x, s) d s$, and $\Omega$ is a bounded domain in $\mathbb{R}^{N}$.

The (AR) condition above is somewhat natural and important to guarantee the boundedness of Palais-Smale sequence of Euler-Lagrange functional for an elliptic equation, however, this condition is very restrictive and eliminates many nonlinearities. Thus, many researchers have tried to drop the (AR) condition for elliptic equations associated with the $p$-Laplacian; see, e.g., $[1,23,25,26,29]$.

The purpose of this paper is to study the existence of weak solutions for problem (P) without assuming the (AR) condition, but imposing various assumptions for the divergence part $\varphi$ and nonlinear term $f$. In particular, as observed by Remark 1.8 in [23], there are many examples which do not fulfill the condition of the nonlinear term $f$ given in $[1,25$, 26]. On the other hand, in case of the whole space $\mathbb{R}^{N}$, the main difficulty of this problem is the lack of compactness for the Sobolev theorem. In that sense, our study is to pursue two goals. First, we show the existence of nontrivial weak solutions for the problem above using the mountain pass theorem. To be precise, we prove the existence of weak solutions for problem (P) under Cerami condition, as a weak version of the Palais-Smale condition. Also, we try to do analysis using the properties of Kirchhoff function $M$ and function $\varphi$. Second, we show the multiplicity of weak solutions to problem $(\mathrm{P})$ via the fountain theorem. To the best of our knowledge, there were no such existence results for our problem in this situation. 


\section{Preliminaries}

In this section, we briefly describe the framework for our problem. We assume that the potential $V \in C\left(\mathbb{R}^{N}\right)$ is a continuous function with

(V) $\inf _{x \in \mathbb{R}^{N}} V(x)>0$, and meas $\left\{x \in \mathbb{R}^{N}: V(x) \leq K\right\}<+\infty$ for all $K \in \mathbb{R}$.

Also, we set $D^{p}\left(\mathbb{R}^{N}\right)=\left\{u \in L^{p_{*}}\left(\mathbb{R}^{N}\right) \mid \Delta u \in L^{p}\left(\mathbb{R}^{N}\right)\right\}$. Thus, we define the function space as follows:

$$
X=\left\{u \in D^{p}\left(\mathbb{R}^{N}\right): \int_{\mathbb{R}^{N}}\left(|\Delta u|^{p}+|\nabla u|^{p}+V(x)|u|^{p}\right) d x<+\infty\right\}
$$

equipped with the norm

$$
\|u\|_{X}^{p}=\|\Delta u\|_{L^{p}\left(\mathbb{R}^{N}\right)}^{p}+\|\nabla u\|_{L^{p}\left(\mathbb{R}^{N}\right)}^{p}+\left\|V^{1 / p} u\right\|_{L^{p}\left(\mathbb{R}^{N}\right)}^{p} .
$$

For our problem, we first assume that $M: \mathbb{R}^{+} \rightarrow \mathbb{R}^{+}$satisfies the following conditions:

(M1) $M \in C\left(\mathbb{R}^{+}\right)$satisfies $\inf _{t \in \mathbb{R}^{+}} M(t) \geq m_{0}>0$, where $m_{0}$ is a constant.

(M2) There exists $\theta \in\left[1, \frac{N}{N-p}\right)$ such that $\theta \mathcal{M}(t)=\theta \int_{0}^{t} M(\tau) d \tau \geq M(t) t$ for any $t \geq 0$.

A typical example for $M$ is given by $M(t)=b_{0}+b_{1} t^{n}$ with $n>0, b_{0}>0$, and $b_{1} \geq 0$.

Next, we assume that $\varphi: \mathbb{R}^{N} \times \mathbb{R}^{N} \rightarrow \mathbb{R}^{N}$ is a continuous function with the continuous derivative with respect to $v$ of the mapping $\Phi_{0}: \mathbb{R}^{N} \times \mathbb{R}^{N} \rightarrow \mathbb{R}, \Phi_{0}=\Phi_{0}(x, v)$, that is, $\varphi(x, v)=\frac{d}{d v} \Phi_{0}(x, v)$. Suppose that $\varphi$ and $\Phi_{0}$ satisfy the following assumptions:

(J1) The equality

$$
\Phi_{0}(x, \mathbf{0})=0
$$

holds for almost all $x \in \mathbb{R}^{N}$.

(J2) There are a nonnegative function $a \in L^{p^{\prime}}\left(\mathbb{R}^{N}\right)$ and a nonnegative constant $b$ such that

$$
|\varphi(x, v)| \leq a(x)+b|v|^{p-1}
$$

holds for almost all $x \in \mathbb{R}^{N}$ and for all $v \in \mathbb{R}^{N}$. Here, $p^{\prime}$ is a conjugate number of $p$.

(J3) The relations

$$
d|v|^{p} \leq \varphi(x, v) \cdot v \quad \text { and } \quad d|v|^{p} \leq p \Phi_{0}(x, v)
$$

hold for all $x \in \mathbb{R}^{N}$ and $v \in \mathbb{R}^{N}$, where $d$ is a positive constant.

(J4) $\Phi_{0}(x, \cdot)$ is strictly convex in $\mathbb{R}^{N}$ for all $x \in \mathbb{R}^{N}$.

(J5) The relation

$$
p \Phi_{0}(x, v)-\varphi(x, v) \cdot v \geq 0
$$

holds for all $x \in \mathbb{R}^{N}$ and all $v \in \mathbb{R}^{N}$.

Let us define the functional $\Phi: X \rightarrow \mathbb{R}$ by

$$
\Phi(u)=\frac{1}{p} \int_{\mathbb{R}^{N}}|\Delta u|^{p} d x+\mathcal{M}\left(\int_{\mathbb{R}^{N}} \Phi_{0}(x, \nabla u) d x\right)+\frac{1}{p} \int_{\mathbb{R}^{N}} V(x)|u|^{p} d x .
$$


It is not difficult to prove that the functional $\Phi \in C^{1}(X, \mathbb{R})$, and its Fréchet derivative is given by

$$
\begin{aligned}
\left\langle\Phi^{\prime}(u), v\right\rangle= & \int_{\mathbb{R}^{N}}|\Delta u|^{p-2} \Delta u \Delta v d x+M\left(\int_{\mathbb{R}^{N}} \Phi_{0}(x, \nabla u) d x\right) \int_{\mathbb{R}^{N}} \varphi(x, \nabla u) \cdot \nabla v d x \\
& +\int_{\mathbb{R}^{N}} V(x)|u|^{p-2} u v d x .
\end{aligned}
$$

We give some examples satisfying assumptions (J1)-(J5).

\section{Example 2.1}

(1) Let us consider the following functions:

$$
\varphi(x, v)=|v|^{p-2} v \quad \text { and } \quad \Phi_{0}(x, v)=\frac{|v|^{p}}{p}
$$

for $v \in \mathbb{R}^{N}$ and $x \in \mathbb{R}^{N}$. Then it is obvious that assumptions (J1)-(J5) hold.

(2) Suppose that $a \in L^{2 p^{\prime}}\left(\mathbb{R}^{N}\right)$, and there is a positive constant $a_{0}$ such that $a(x) \geq a_{0}$ for almost all $x \in \mathbb{R}^{N}$. We consider

$$
\varphi(x, t)=\left(a(x)+t^{2}\right)^{\frac{p-2}{2}} t \quad \text { and } \quad \Phi_{0}(x, t)=\frac{1}{p}\left[\left(a(x)+t^{2}\right)^{\frac{p}{2}}-a(x)^{\frac{p}{2}}\right]
$$

for $t \in \mathbb{R}$, where $p \geq 2$ for all $x \in \mathbb{R}^{N}$. Then assumptions (J1)-(J5) hold.

By analogous arguments as in $[19,22]$, the following lemma is easily checked, and thus we omit the proof. That is, the operator $\Phi^{\prime}$ is a mapping of type $\left(S_{+}\right)$.

Lemma 2.2 Assume that (V), (M1), (M2), and (J1)-(J4) hold. Then the functional $\Phi$ : $X \rightarrow \mathbb{R}$ is convex and weakly lower semicontinuous on $X$. Moreover, the operator $\Phi^{\prime}$ is a mapping of type $\left(S_{+}\right)$, i.e., if $u_{n} \rightarrow u$ in $X$ and $\lim \sup _{n \rightarrow \infty}\left\langle\Phi^{\prime}\left(u_{n}\right)-\Phi^{\prime}(u), u_{n}-u\right\rangle \leq 0$, then $u_{n} \rightarrow$ in $X$ as $n \rightarrow \infty$.

Denoting $F(x, t)=\int_{0}^{t} f(x, s) d s$, for the number $\theta$ given in (M2), we assume that

(F1) $f: \mathbb{R}^{N} \times \mathbb{R} \rightarrow \mathbb{R}$ satisfies the Carathéodory condition in the sense that $f(\cdot, t)$ is measurable for all $t \in \mathbb{R}$ and $f(x, \cdot)$ is continuous for almost all $x \in \mathbb{R}^{N}$.

(F2) There exist nonnegative functions $\rho \in L^{q^{\prime}}\left(\mathbb{R}^{N}\right) \cap L^{\infty}\left(\mathbb{R}^{N}\right)$ and $\sigma \in L^{\infty}\left(\mathbb{R}^{N}\right)$ such that

$$
|f(x, t)| \leq \rho(x)+\sigma(x)|t|^{q-1}, \quad q \in\left(\theta p, p_{*}\right)
$$

for all $(x, t) \in \mathbb{R}^{N} \times \mathbb{R}$.

(F3) There exists $\delta>0$ such that

$$
F(x, t) \leq 0 \quad \text { for } x \in \mathbb{R}^{N} \text { and }|t|<\delta .
$$

(F4) $\lim _{|t| \rightarrow \infty} \frac{F(x, t)}{|t|^{\theta p}}=\infty$ uniformly for almost all $x \in \mathbb{R}^{N}$. 
(F5) There exist $c_{0} \geq 0, r_{0} \geq 0$, and $\kappa>\frac{N}{p}$ such that

$$
|F(x, t)|^{\kappa} \leq c_{0}|t|^{\kappa p} \mathfrak{F}(x, t)
$$

for all $(x, t) \in \mathbb{R}^{N} \times \mathbb{R}$ and $|t| \geq r_{0}$, where $\mathfrak{F}(x, t)=\frac{1}{\theta p} f(x, t) t-F(x, t) \geq 0$.

(F6) There exist $\mu>\theta p$ and $\varrho>0$ such that

$$
\mu F(x, t) \leq t f(x, t)+\varrho t^{p}
$$

for all $(x, t) \in \mathbb{R}^{N} \times \mathbb{R}$.

Next, we give some examples with respect to assumptions (F1)-(F6).

Since assumption (F5) is weaker than the following assumption, namely that

$$
\frac{f(x, t)}{|t|^{\theta-2} t} \text { is increasing for } t>0 \text { and decreasing for } t<0
$$

for any $x \in \mathbb{R}^{N}$, we check that the following example satisfies assumption (F5) by applying condition (2.1).

Example 2.3 Let us consider

$$
f(x, t)=|t|^{q-2} t \log (1+|t|)
$$

for all $t \in \mathbb{R}$. It is clear that function $f$ satisfies assumptions (F1)-(F4). Since the following ratio, namely

$$
\frac{f(x, t)}{|t|^{p-2} t}=\frac{|t|^{q-2} t \log (1+|t|)}{|t|^{p-2} t}=|t|^{q-p} \log (1+|t|)
$$

is increasing for $t>0$ and decreasing for $t<0$ if $q>p=\theta$, it follows that assumption (F5) holds.

The following example can be found in [23] for the case of $p$-Laplace operator.

Example 2.4 Consider the following function:

$$
f(x, t)=|t|^{p-2} t\left(4|t|^{3}+2 t \sin t-4 \cos t\right) .
$$

Then this function satisfies conditions (F2), (F6), but not the (AR) condition.

Define the functional $\Psi: X \rightarrow \mathbb{R}$ by

$$
\Psi(u)=\int_{\mathbb{R}^{N}} F(x, u) d x
$$

Then it is easy to check that $\Psi \in C^{1}(X, \mathbb{R})$ and its Fréchet derivative is

$$
\left\langle\Psi^{\prime}(u), v\right\rangle=\int_{\mathbb{R}^{N}} f(x, u) v d x
$$


for any $u, v \in X$. Next we define the functional $I_{\lambda}: X \rightarrow \mathbb{R}$ by

$$
I_{\lambda}(u)=\Phi(u)-\lambda \Psi(u)
$$

Then it follows that the functional $I_{\lambda} \in C^{1}(X, \mathbb{R})$ and its Fréchet derivative is

$$
\begin{aligned}
\left\langle I_{\lambda}^{\prime}(u), v\right\rangle= & \int_{\mathbb{R}^{N}}|\Delta u|^{p-2} \Delta u \Delta v d x+M\left(\int_{\mathbb{R}^{N}} \Phi_{0}(x, \nabla u) d x\right) \int_{\mathbb{R}^{N}} \varphi(x, \nabla u) \cdot \nabla v d x \\
& +\int_{\mathbb{R}^{N}} V(x)|u|^{p-2} u v d x-\lambda \int_{\mathbb{R}^{N}} f(x, u) v d x
\end{aligned}
$$

for any $u, v \in X$.

In our setting, first of all, we need the following lemma. Using a similar argument as in [17, Lemma 3.2], we can see that the functionals $\Psi$ and $\Psi^{\prime}$ are weakly strongly continuous on $X$. We give a detailed proof for the convenience of the reader.

Lemma 2.5 Assume that (V) and (F1)-(F2) hold. Then $\Psi$ and $\Psi^{\prime}$ are weakly strongly continuous on $X$.

Proof See Appendix.

\section{Existence of weak solutions}

Definition 3.1 We say that $u \in X$ is a weak solution of problem (P) if

$$
\begin{gathered}
\int_{\mathbb{R}^{N}}|\Delta u|^{p-2} \Delta u \cdot \Delta v d x+M\left(\int_{\mathbb{R}^{N}} \Phi_{0}(x, \nabla u) d x\right) \int_{\mathbb{R}^{N}} \varphi(x, \nabla u) \cdot \nabla v d x \\
\quad+\int_{\mathbb{R}^{N}} V(x)|u|^{p-2} u v d x-\lambda \int_{\mathbb{R}^{N}} f(x, u) v d x=0
\end{gathered}
$$

for any $v \in X$.

The following result is used to show that the energy functional $I_{\lambda}$ satisfies the geometric conditions of the mountain pass theorem.

Lemma 3.2 Assume that (V), (M1), (M2), (J1)-(J3), and (F1)-(F4) hold. Then the geometric conditions in the mountain pass theorem hold, i.e.,

(1) $u=0$ is a strict local minimum for $I_{\lambda}(u)$,

(2) $I_{\lambda}(u)$ is unbounded from below on $X$.

Proof By assumption (F3), $u=0$ is a strict local minimum for $I_{\lambda}(u)$. Next we claim that condition (2) holds. Assumption (F4) implies that for any $K_{0}>0$, there exists a constant $\delta>0$ such that

$$
F(x, t) \geq K_{0}|t|^{\theta p}
$$


for $|t|>\delta$ and for almost all $x \in \mathbb{R}^{N}$. Note that for $t>1$, we can easily check that $\mathcal{M}(t) \leq$ $\mathcal{M}(1) t$. For any $v \in X \backslash\{0\}$, from assumptions (J2), (J3) and relation (3.1), we have

$$
\begin{aligned}
I_{\lambda}(t v)= & \frac{1}{p} \int_{\mathbb{R}^{N}}|t \Delta v|^{p} d x+\mathcal{M}\left(\int_{\mathbb{R}^{N}} \Phi_{0}(x, t \nabla v) d x\right) \\
& +\frac{1}{p} \int_{\mathbb{R}^{N}} V(x)|t|^{p}|v|^{p} d x-\lambda \int_{\mathbb{R}^{N}} F(x, t v) d x \\
\leq & \frac{1}{p} \int_{\mathbb{R}^{N}}|t \Delta v|^{p} d x+\mathcal{M}(1)\left(\int_{\mathbb{R}^{N}} \Phi_{0}(x, t \nabla v) d x\right)^{\theta} \\
& +\frac{1}{p} \int_{\mathbb{R}^{N}} V(x)|t|^{p}|v|^{p} d x-\lambda \int_{\mathbb{R}^{N}} F(x, t v) d x \\
\leq & \frac{1}{p}|t|^{p}\|v\|_{X}^{p}+\mathcal{M}(1)\left(\int_{\mathbb{R}^{N}} a(x)|t \nabla v|+\frac{b}{p}|t \nabla v|^{p} d x\right)^{\theta}-\lambda \int_{\mathbb{R}^{N}} F(x, t v) d x \\
\leq & |t|^{\theta p}\left(\frac{1}{p}\|v\|_{X}^{p}+\mathcal{M}(1)\left(\int_{\mathbb{R}^{N}} a(x)|\nabla v|+\frac{b}{p}|\nabla v|^{p} d x\right)^{\theta}-\lambda K_{0} \int_{\mathbb{R}^{N}}|v|^{\theta p} d x\right)
\end{aligned}
$$

for sufficiently large $t>1$. If $K_{0}$ is large enough, then we assert that $I_{\lambda}(t v) \rightarrow-\infty$ as $t \rightarrow \infty$. Hence we conclude that the functional $I_{\lambda}$ is unbounded from below. This completes the proof.

With the aid of Lemmas 2.2 and 2.5, we prove that the energy functional $I_{\lambda}$ satisfies the Cerami condition $(C)_{c}$ condition, for short, i.e., for $c \in \mathbb{R}$, any sequence $\left\{u_{n}\right\} \subset X$ such that

$$
I_{\lambda}\left(u_{n}\right) \rightarrow c \quad \text { and } \quad\left\|I_{\lambda}^{\prime}\left(u_{n}\right)\right\|_{X^{*}}\left(1+\left\|u_{n}\right\|_{X}\right) \rightarrow 0 \quad \text { as } n \rightarrow \infty
$$

has a convergent subsequence. This plays a key role in obtaining the existence of a nontrivial weak solution for the given problem.

Lemma 3.3 Assume that (V), (M1), (M2), (J1)-(J5), and (F1)-(F5) hold. Then the functional $I_{\lambda}$ satisfies the $(C)_{c}$ condition for any $\lambda>0$.

Proof For $c \in \mathbb{R}$, let $\left\{u_{n}\right\}$ be a $(C)_{c}$-sequence in $X$, that is,

$$
I_{\lambda}\left(u_{n}\right) \rightarrow c \quad \text { and } \quad\left\|I_{\lambda}^{\prime}\left(u_{n}\right)\right\|_{X^{*}}\left(1+\left\|u_{n}\right\|_{X}\right) \rightarrow 0 \quad \text { as } n \rightarrow \infty
$$

This says that

$$
c=I_{\lambda}\left(u_{n}\right)+o(1) \text { and }\left\langle I_{\lambda}^{\prime}\left(u_{n}\right), u_{n}\right\rangle=o(1),
$$

where $o(1) \rightarrow 0$ as $n \rightarrow \infty$. It follows from Lemmas 2.2 and 2.5 that $\Phi^{\prime}$ and $\Psi^{\prime}$ are mappings of type $\left(S_{+}\right)$. Since $I_{\lambda}^{\prime}$ is of type $\left(S_{+}\right)$and $X$ is reflexive, it suffices to prove that the sequence $\left\{u_{n}\right\}$ is bounded in $X$. We argue by contradiction. Suppose that the sequence $\left\{u_{n}\right\}$ is unbounded in $X$. Then we may assume that $\left\|u_{n}\right\|_{X}>1$ and $\left\|u_{n}\right\|_{X} \rightarrow \infty$ as $n \rightarrow \infty$. Define a sequence $\left\{w_{n}\right\}$ by $w_{n}=u_{n} /\left\|u_{n}\right\|_{X}$. It is clear that $\left\{w_{n}\right\} \subset X$ and $\left\|w_{n}\right\|_{X}=1$. Hence, up to a subsequence still denoted by $\left\{w_{n}\right\}$, we obtain $w_{n} \rightarrow w$ in $X$ as $n \rightarrow \infty$ and note that

$$
w_{n}(x) \rightarrow w(x) \quad \text { a.e. in } \mathbb{R}^{N} \quad \text { and } \quad w_{n} \rightarrow w \quad \text { in } L^{s}\left(\mathbb{R}^{N}\right) \text { as } n \rightarrow \infty
$$


for $1<s<p_{*}$. According to assumptions (M1), (M2), (J3), and relation (3.3), we obtain that

$$
\begin{aligned}
c= & I_{\lambda}\left(u_{n}\right)+o(1) \\
= & \frac{1}{p} \int_{\mathbb{R}^{N}}\left|\Delta u_{n}\right|^{p} d x+\mathcal{M}\left(\int_{\mathbb{R}^{N}} \Phi_{0}\left(x, \nabla u_{n}\right) d x\right) \\
& +\frac{1}{p} \int_{\mathbb{R}^{N}} V(x)\left|u_{n}\right|^{p} d x-\lambda \int_{\mathbb{R}^{N}} F\left(x, u_{n}\right) d x+o(1) \\
\geq & \frac{1}{p} \int_{\mathbb{R}^{N}}\left|\Delta u_{n}\right|^{p} d x+\frac{1}{\theta} M\left(\int_{\mathbb{R}^{N}} \Phi_{0}\left(x, \nabla u_{n}\right) d x\right) \int_{\mathbb{R}^{N}} \Phi_{0}\left(x, \nabla u_{n}\right) d x \\
& +\frac{1}{p} \int_{\mathbb{R}^{N}} V(x)\left|u_{n}\right|^{p} d x-\lambda \int_{\mathbb{R}^{N}} F\left(x, u_{n}\right) d x+o(1) \\
\geq & \frac{1}{p} \int_{\mathbb{R}^{N}}\left|\Delta u_{n}\right|^{p} d x+\frac{d m_{0}}{\theta p} \int_{\mathbb{R}^{N}}\left|\nabla u_{n}\right|^{p} d x \\
& +\frac{1}{p} \int_{\mathbb{R}^{N}} V(x)\left|u_{n}\right|^{p} d x-\lambda \int_{\mathbb{R}^{N}} F\left(x, u_{n}\right) d x+o(1) \\
\geq & \frac{\min \left\{1, d m_{0}\right\}}{\theta p}\left\|u_{n}\right\|_{X}^{p}-\lambda \int_{\mathbb{R}^{N}} F\left(x, u_{n}\right) d x+o(1) .
\end{aligned}
$$

Since $\left\|u_{n}\right\|_{X} \rightarrow \infty$ as $n \rightarrow \infty$, we have

$$
\int_{\mathbb{R}^{N}} F\left(x, u_{n}\right) d x \geq \frac{\min \left\{1, d m_{0}\right\}}{\theta p \lambda}\left\|u_{n}\right\|_{X}^{p}-\frac{c}{\lambda}+\frac{o(1)}{\lambda} \rightarrow \infty \quad \text { as } n \rightarrow \infty .
$$

In addition, we assert that

$$
\begin{aligned}
I_{\lambda}\left(u_{n}\right)= & \frac{1}{p} \int_{\mathbb{R}^{N}}\left|\Delta u_{n}\right|^{p} d x+\mathcal{M}\left(\int_{\mathbb{R}^{N}} \Phi_{0}\left(x, \nabla u_{n}\right) d x\right) \\
& +\frac{1}{p} \int_{\mathbb{R}^{N}} V(x)\left|u_{n}\right|^{p} d x-\lambda \int_{\mathbb{R}^{N}} F\left(x, u_{n}\right) d x \\
\leq & \frac{1}{p}\left\|u_{n}\right\|_{X}^{p}+\mathcal{M}\left(\int_{\mathbb{R}^{N}} \Phi_{0}\left(x, \nabla u_{n}\right) d x\right)-\lambda \int_{\mathbb{R}^{N}} F\left(x, u_{n}\right) d x .
\end{aligned}
$$

Combining this with relation (3.3), we obtain that

$$
\frac{1}{p}\left\|u_{n}\right\|_{X}^{p}+\mathcal{M}\left(\int_{\mathbb{R}^{N}} \Phi_{0}\left(x, \nabla u_{n}\right) d x\right) \geq \lambda \int_{\mathbb{R}^{N}} F\left(x, u_{n}\right) d x+c-o(1)
$$

for sufficiently large $n$. Assumption (F4) implies that there exists $t_{0}>1$ such that $F(x, t)>$ $|t|^{\theta p}$ for all $x \in \mathbb{R}^{N}$ and $|t|>t_{0}$. From assumptions (F1) and (F2), there exists $\mathcal{C}>0$ such that $|F(x, t)| \leq \mathcal{C}$ for all $(x, t) \in \mathbb{R}^{N} \times\left[-t_{0}, t_{0}\right]$. Therefore we can choose a real number $\mathcal{C}_{0}$ such that $F(x, t) \geq \mathcal{C}_{0}$ for all $(x, t) \in \mathbb{R}^{N} \times \mathbb{R}$, and thus

$$
\frac{F\left(x, u_{n}\right)-\mathcal{C}_{0}}{\frac{1}{p}\left\|u_{n}\right\|_{X}^{p}+\mathcal{M}\left(\int_{\mathbb{R}^{N}} \Phi_{0}\left(x, \nabla u_{n}\right) d x\right)} \geq 0
$$

for all $x \in \mathbb{R}^{N}$ and for all $n \in \mathbb{N}$. Set $\Omega_{1}=\left\{x \in \mathbb{R}^{N}: w(x) \neq 0\right\}$. By the convergence in (3.4), we know that

$$
\left|u_{n}(x)\right|=\left|w_{n}(x)\right|\left\|u_{n}\right\|_{X} \rightarrow \infty \quad \text { as } n \rightarrow \infty
$$


for all $x \in \Omega_{1}$. So then, it follows from assumptions (M2), (J2), (F4), and Hölder's inequality that, for all $x \in \Omega_{1}$, we have

$$
\begin{aligned}
& \lim _{n \rightarrow \infty} \frac{F\left(x, u_{n}\right)}{\frac{1}{p}\left\|u_{n}\right\|_{X}^{p}+\mathcal{M}\left(\int_{\mathbb{R}^{N}} \Phi_{0}\left(x, \nabla u_{n}\right) d x\right)} \\
& \quad \geq \lim _{n \rightarrow \infty} \frac{F\left(x, u_{n}\right)}{\frac{1}{p}\left\|u_{n}\right\|_{X}^{p}+\mathcal{M}(1)\left(1+\left(\int_{\mathbb{R}^{N}} \Phi_{0}\left(x, \nabla u_{n}\right) d x\right)^{\theta}\right)} \\
& \quad \geq \lim _{n \rightarrow \infty} \frac{F\left(x, u_{n}\right)}{\frac{1}{p}\left\|u_{n}\right\|_{X}^{p}+\mathcal{M}(1)\left(1+\left(\int_{\mathbb{R}^{N}} a(x)\left|\nabla u_{n}\right|+\frac{b}{p}\left|\nabla u_{n}\right|^{p} d x\right)^{\theta}\right)} \\
& \quad \geq \lim _{n \rightarrow \infty} \frac{F\left(x, u_{n}\right)}{\frac{1}{p}\left\|u_{n}\right\|_{X}^{p}+\mathcal{M}(1)\left(1+\left(\|a\|_{L^{p^{\prime}}\left(\mathbb{R}^{N}\right)}^{p}\left\|\nabla u_{n}\right\|_{L^{p}\left(\mathbb{R}^{N}\right)}+\frac{b}{p}\left\|\nabla u_{n}\right\|_{\left.L^{p}\left(\mathbb{R}^{N}\right)^{\theta}\right)}^{\theta^{\prime}}\right.\right.} \\
& \quad \geq \lim _{n \rightarrow \infty} \frac{F\left(x, u_{n}\right)}{\left(\frac{1}{p}+\mathcal{M}(1)\left(1+\left(\|a\|_{L^{p^{\prime}}\left(\mathbb{R}^{N}\right)}+\frac{b}{p}\right) \theta^{\theta}\right)\right)\left\|u_{n}\right\|_{X}^{\theta p}} \\
& \quad \lim _{n \rightarrow \infty} \frac{F\left(x, u_{n}\right)}{\left(\frac{1}{p}+\mathcal{M}(1)\left(1+\left(\|a\|_{L^{p^{\prime}}\left(\mathbb{R}^{N}\right)}+\frac{b}{p}\right)^{\theta}\right)\right)\left|u_{n}(x)\right|^{\theta p}}\left|w_{n}(x)\right|^{\theta p} \\
& \quad=\infty,
\end{aligned}
$$

where we have used the inequality $\mathcal{M}(t) \leq \mathcal{M}(1)\left(1+t^{\theta}\right)$ for all $t \in \mathbb{R}^{+}$, since if $0 \leq t<1$, then $\mathcal{M}(t)=\int_{0}^{t} M(\tau) d \tau \leq \mathcal{M}(1)$ and if $t>1$, then $\mathcal{M}(t) \leq \mathcal{M}(1) t^{\theta}$. Hence we get that meas $\left(\Omega_{1}\right)=0$. Indeed, if meas $\left(\Omega_{1}\right) \neq 0$, according to (3.6)-(3.8) and Fatou's lemma, we would obtain

$$
\begin{aligned}
\frac{1}{\lambda}= & \liminf _{n \rightarrow \infty} \frac{\int_{\mathbb{R}^{N}} F\left(x, u_{n}\right) d x}{\lambda \int_{\mathbb{R}^{N}} F\left(x, u_{n}\right) d x+c-o(1)} \\
\geq & \liminf _{n \rightarrow \infty} \int_{\mathbb{R}^{N}} \frac{F\left(x, u_{n}\right)}{\frac{1}{p}\left\|u_{n}\right\|_{X}^{p}+\mathcal{M}\left(\int_{\mathbb{R}^{N}} \Phi_{0}\left(x, \nabla u_{n}\right) d x\right)} d x \\
\geq & \liminf _{n \rightarrow \infty} \int_{\Omega_{1}} \frac{F\left(x, u_{n}\right)}{\frac{1}{p}\left\|u_{n}\right\|_{X}^{p}+\mathcal{M}\left(\int_{\mathbb{R}^{N}} \Phi_{0}\left(x, \nabla u_{n}\right) d x\right)} d x \\
& -\limsup _{n \rightarrow \infty} \int_{\Omega_{1}} \frac{\mathcal{C}_{0}}{\frac{1}{p}\left\|u_{n}\right\|_{X}^{p}+\mathcal{M}\left(\int_{\mathbb{R}^{N}} \Phi_{0}\left(x, \nabla u_{n}\right) d x\right)} d x \\
= & \liminf _{n \rightarrow \infty} \int_{\Omega_{1}} \frac{F\left(x, u_{n}\right)-\mathcal{C}_{0}}{\frac{1}{p}\left\|u_{n}\right\|_{X}^{p}+\mathcal{M}\left(\int_{\mathbb{R}^{N}} \Phi_{0}\left(x, \nabla u_{n}\right) d x\right)} d x \\
\geq & \int_{\Omega_{1}} \liminf _{n \rightarrow \infty} \frac{F\left(x, u_{n}\right)-\mathcal{C}_{0}}{\frac{1}{p}\left\|u_{n}\right\|_{X}^{p}+\mathcal{M}\left(\int_{\mathbb{R}^{N}} \Phi_{0}\left(x, \nabla u_{n}\right) d x\right)} \frac{F\left(x, u_{n}\right)}{\frac{1}{p}\left\|u_{n}\right\|_{X}^{p}+\mathcal{M}\left(\int_{\mathbb{R}^{N}} \Phi_{0}\left(x, \nabla u_{n}\right) d x\right)} d x \\
& -\int_{\Omega_{1}} \limsup _{n \rightarrow \infty} \frac{\mathcal{C}_{0}}{\frac{1}{p}\left\|u_{n}\right\|_{X}^{p}+\mathcal{M}\left(\int_{\mathbb{R}^{N}} \Phi_{0}\left(x, \nabla u_{n}\right) d x\right)} d x \\
= & \infty, \quad
\end{aligned}
$$


which is a contradiction. Thus $w(x)=0$ for almost all $x \in \mathbb{R}^{N}$. Using assumptions (M1)(M2) and (J5), we get

$$
\begin{aligned}
c+1 \geq & I_{\lambda}\left(u_{n}\right)-\frac{1}{\theta p}\left\langle I_{\lambda}^{\prime}\left(u_{n}\right), u_{n}\right\rangle \\
= & \frac{1}{p} \int_{\mathbb{R}^{N}}\left|\Delta u_{n}\right|^{p} d x+\mathcal{M}\left(\int_{\mathbb{R}^{N}} \Phi_{0}\left(x, \nabla u_{n}\right) d x\right) \\
& +\frac{1}{p} \int_{\mathbb{R}^{N}} V(x)\left|u_{n}\right|^{p} d x-\lambda \int_{\mathbb{R}^{N}} F\left(x, u_{n}\right) d x \\
& -\frac{1}{\theta p} \int_{\mathbb{R}^{N}}\left|\Delta u_{n}\right|^{p} d x-\frac{1}{\theta p} M\left(\int_{\mathbb{R}^{N}} \Phi_{0}\left(x, \nabla u_{n}\right) d x\right) \int_{\mathbb{R}^{N}} \varphi\left(x, \nabla u_{n}\right) \cdot \nabla u_{n} d x \\
& -\frac{1}{\theta p} \int_{\mathbb{R}^{N}} V(x)\left|u_{n}\right|^{p} d x+\frac{1}{\theta p} \lambda \int_{\mathbb{R}^{N}} f\left(x, u_{n}\right) u_{n} d x \\
\geq & \frac{1}{\theta} M\left(\int_{\mathbb{R}^{N}} \Phi_{0}\left(x, \nabla u_{n}\right) d x\right) \int_{\mathbb{R}^{N}} \Phi_{0}\left(x, \nabla u_{n}\right) d x-\lambda \int_{\mathbb{R}^{N}} F\left(x, u_{n}\right) d x \\
& -\frac{1}{\theta p} M\left(\int_{\mathbb{R}^{N}} \Phi_{0}\left(x, \nabla u_{n}\right) d x\right) \int_{\mathbb{R}^{N}} \varphi\left(x, \nabla u_{n}\right) \cdot \nabla u_{n} d x+\frac{1}{\theta p} \lambda \int_{\mathbb{R}^{N}} f\left(x, u_{n}\right) u_{n} d x \\
= & \frac{1}{\theta} M\left(\int_{\mathbb{R}^{N}} \Phi_{0}\left(x, \nabla u_{n}\right) d x\right)\left(\int_{\mathbb{R}^{N}} \Phi_{0}\left(x, \nabla u_{n}\right) d x-\frac{1}{p} \int_{\mathbb{R}^{N}} \varphi\left(x, \nabla u_{n}\right) \cdot \nabla u_{n} d x\right) \\
& +\lambda \int_{\mathbb{R}^{N}} \mathfrak{F}\left(x, u_{n}\right) d x \\
\geq & \lambda \int_{\mathbb{R}^{N}} \mathfrak{F}\left(x, u_{n}\right) d x
\end{aligned}
$$

for $n$ large enough. Let us define $\Omega_{n}(a, b):=\left\{x \in \mathbb{R}^{N}: a \leq\left|u_{n}(x)\right|<b\right\}$ for $a \geq 0$. The convergence in (3.4) means that

$$
w_{n} \rightarrow 0 \quad \text { in } L^{r}\left(\mathbb{R}^{N}\right) \quad \text { and } \quad w_{n}(x) \rightarrow 0 \quad \text { a.e. in } \mathbb{R}^{N} \text { as } n \rightarrow \infty
$$

for $1<r<p_{*}$. Hence by using (3.5) we get

$$
0<\frac{\min \left\{1, d m_{0}\right\}}{\lambda \theta p} \leq \limsup _{n \rightarrow \infty} \int_{\mathbb{R}^{N}} \frac{\left|F\left(x, u_{n}\right)\right|}{\left\|u_{n}\right\|_{X}^{p}} d x
$$

On the other hand, from assumption (F2) and relation (3.11), it follows that

$$
\begin{aligned}
& \int_{\Omega_{n}\left(0, r_{0}\right)} \frac{F\left(x, u_{n}\right)}{\left\|u_{n}\right\|_{X}^{p}} d x \\
& \leq \int_{\Omega_{n}\left(0, r_{0}\right)} \frac{\rho(x)\left|u_{n}(x)\right|+\frac{\sigma(x)}{q}\left|u_{n}(x)\right|^{q}}{\left\|u_{n}\right\|_{X}^{p}} d x \\
& \leq \frac{C_{1}}{\left\|u_{n}\right\|_{X}^{p}}\|\rho\|_{L^{q^{\prime}}\left(\mathbb{R}^{N}\right)}\left\|u_{n}\right\|_{L^{q}\left(\mathbb{R}^{N}\right)} \\
& \quad+\frac{\|\sigma\|_{L^{\infty}\left(\mathbb{R}^{N}\right)}}{q} \int_{\Omega_{n}\left(0, r_{0}\right)}\left|u_{n}(x)\right|^{q-p}\left|w_{n}(x)\right|^{p} d x \\
& \leq \frac{C_{1}}{\left\|u_{n}\right\|_{X}^{p}}\|\rho\|_{L^{q^{\prime}}\left(\mathbb{R}^{N}\right)}\left\|u_{n}\right\|_{L^{q}\left(\mathbb{R}^{N}\right)}+\frac{\|\sigma\|_{L^{\infty}\left(\mathbb{R}^{N}\right)}}{q} r_{0}^{q-p} \int_{\mathbb{R}^{N}}\left|w_{n}(x)\right|^{p} d x
\end{aligned}
$$




$$
\begin{aligned}
& \leq \frac{C_{2}}{\left\|u_{n}\right\|_{X}^{p}}\|\rho\|_{L^{q^{\prime}}\left(\mathbb{R}^{N}\right)}\left\|u_{n}\right\|_{X}+\frac{\|\sigma\|_{L^{\infty}\left(\mathbb{R}^{N}\right)}}{q} r_{0}^{q-p} \int_{\mathbb{R}^{N}}\left|w_{n}(x)\right|^{p} d x \\
& \leq \frac{C_{3}}{\left\|u_{n}\right\|_{X}^{p-1}}+\frac{\|\sigma\|_{L^{\infty}\left(\mathbb{R}^{N}\right)}}{q} r_{0}^{q-p} \int_{\mathbb{R}^{N}}\left|w_{n}(x)\right|^{p} d x \rightarrow 0 \quad \text { as } n \rightarrow \infty
\end{aligned}
$$

for some positive constants $C_{i}(i=1,2,3)$. Set $\kappa^{\prime}=\kappa /(\kappa-1)$. Since $\kappa>N / p$, we get $1<\kappa^{\prime} p<$ $p_{*}$. Hence, it follows from (F5), (3.10), and (3.11) that

$$
\begin{aligned}
\int_{\Omega_{n}\left(r_{0}, \infty\right)} \frac{\left|F\left(x, u_{n}\right)\right|}{\left\|u_{n}\right\|_{X}^{p}} d x & \leq \int_{\Omega_{n}\left(r_{0}, \infty\right)} \frac{\left|F\left(x, u_{n}\right)\right|}{\left|u_{n}(x)\right|^{p}}\left|w_{n}(x)\right|^{p} d x \\
& \leq\left\{\int_{\Omega_{n}\left(r_{0}, \infty\right)}\left(\frac{\left|F\left(x, u_{n}\right)\right|}{\left|u_{n}(x)\right|^{p}}\right)^{\kappa} d x\right\}^{\frac{1}{\kappa}}\left\{\int_{\Omega\left(r_{0}, \infty\right)}\left|w_{n}(x)\right|^{\kappa^{\prime} p}\right\}^{\frac{1}{\kappa^{\prime}}} \\
& \leq c_{0}^{\frac{1}{\kappa}}\left\{\int_{\Omega_{n}\left(r_{0}, \infty\right)} \mathfrak{F}\left(x, u_{n}\right) d x\right\}^{\frac{1}{\kappa}}\left\{\int_{\mathbb{R}^{N}}\left|w_{n}(x)\right|^{\kappa^{\prime} p}\right\}^{\frac{1}{\kappa^{\prime}}} \\
& \leq c_{0}^{\frac{1}{\kappa}}\left(\frac{c+1}{\lambda}\right)^{\frac{1}{\kappa}}\left\{\int_{\mathbb{R}^{N}}\left|w_{n}(x)\right|^{\kappa^{\prime} p}\right\}^{\frac{1}{\kappa^{\prime}}} \rightarrow 0 \text { as } n \rightarrow \infty .
\end{aligned}
$$

Combining the estimates in (3.13) with (3.14), we have

$$
\int_{\mathbb{R}^{N}} \frac{\left|F\left(x, u_{n}\right)\right|}{\left\|u_{n}\right\|_{X}^{p}} d x=\int_{\Omega_{n}\left(0, r_{0}\right)} \frac{\left|F\left(x, u_{n}\right)\right|}{\left\|u_{n}\right\|_{X}^{p}} d x+\int_{\Omega_{n}\left(r_{0}, \infty\right)} \frac{\left|F\left(x, u_{n}\right)\right|}{\left\|u_{n}\right\|_{X}^{p}} d x \rightarrow 0 \quad \text { as } n \rightarrow \infty,
$$

which contradicts (3.12). This completes the proof.

Using Lemma 3.3, we prove the existence of a nontrivial weak solution for our problem under the considered assumptions.

Theorem 3.4 Assume that (V), (M1), (M2), (J1)-(J5), and (F1)-(F5) hold. Then problem (P) has a nontrivial weak solution for all $\lambda>0$.

Proof Note that $I_{\lambda}(0)=0$. In view of Lemma 3.2, the geometric conditions in the mountain pass theorem are fulfilled. And also $I_{\lambda}$ satisfies the $(C)_{c}$ condition for any $\lambda>0$ by Lemma 3.3. Hence, problem $(\mathrm{P})$ has a nontrivial weak solution for all $\lambda>0$. This completes the proof.

Next, under assumption (F6) instead of (F5), we show that $I_{\lambda}$ satisfies the Cerami condition.

Lemma 3.5 Assume that (V), (M1), (M2), (J1)-(J5), (F1)-(F4), and (F6) hold. Then the functional $I_{\lambda}$ satisfies the $(C)_{c}$ condition for any $\lambda>0$.

Proof For $c \in \mathbb{R}$, let $\left\{u_{n}\right\}$ be a $(C)_{c}$-sequence in $X$ satisfying (3.2). Following the proof of Lemma 3.3, we only prove that $\left\{u_{n}\right\}$ is bounded in $X$. To this end, arguing by contradiction, suppose that $\left\|u_{n}\right\|_{X} \rightarrow \infty$ as $n \rightarrow \infty$. Let $v_{n}=u_{n} /\left\|u_{n}\right\|_{X}$. Then $\left\|v_{n}\right\|_{X}=1$. Passing to a subsequence, we may assume that $v_{n} \rightarrow v$ as $n \rightarrow \infty$ in $X$. Thus by an embedding theorem, for $1<s<p_{*}$, we have

$$
v_{n} \rightarrow v \quad \text { in } L^{s}\left(\mathbb{R}^{N}\right) \quad \text { and } \quad v_{n}(x) \rightarrow v(x) \quad \text { a.e. in } \mathbb{R}^{N} \text { as } n \rightarrow \infty .
$$


From (M1), (M2), (J5), and (F6), it follows that

$$
\begin{aligned}
& c+1 \geq I_{\lambda}\left(u_{n}\right)-\frac{1}{\mu}\left\langle I_{\lambda}^{\prime}\left(u_{n}\right), u_{n}\right\rangle \\
& =\frac{1}{p} \int_{\mathbb{R}^{N}}\left|\Delta u_{n}\right|^{p} d x+\mathcal{M}\left(\int_{\mathbb{R}^{N}} \Phi_{0}\left(x, \nabla u_{n}\right) d x\right) \\
& +\frac{1}{p} \int_{\mathbb{R}^{N}} V(x)\left|u_{n}\right|^{p} d x-\lambda \int_{\mathbb{R}^{N}} F\left(x, u_{n}\right) d x \\
& -\frac{1}{\mu} \int_{\mathbb{R}^{N}}\left|\Delta u_{n}\right|^{p} d x-\frac{1}{\mu} M\left(\int_{\mathbb{R}^{N}} \Phi_{0}\left(x, \nabla u_{n}\right) d x\right) \int_{\mathbb{R}^{N}} \varphi\left(x, \nabla u_{n}\right) \cdot \nabla u_{n} d x \\
& -\frac{1}{\mu} \int_{\mathbb{R}^{N}} V(x)\left|u_{n}\right|^{p} d x+\frac{\lambda}{\mu} \int_{\mathbb{R}^{N}} f\left(x, u_{n}\right) u_{n} d x \\
& \geq\left(\frac{1}{p}-\frac{1}{\mu}\right) \int_{\mathbb{R}^{N}}\left|\Delta u_{n}\right|^{p} d x+m_{0}\left(\frac{1}{\theta p}-\frac{1}{\mu}\right) \int_{\mathbb{R}^{N}} \varphi\left(x, \nabla u_{n}\right) \cdot \nabla u_{n} d x \\
& +\left(\frac{1}{p}-\frac{1}{\mu}\right) \int_{\mathbb{R}^{N}} V(x)\left|u_{n}\right|^{p} d x-\frac{\lambda \varrho}{\mu} \int_{\mathbb{R}^{N}}\left|u_{n}\right|^{p} d x \\
& \geq \min \left\{d m_{0}, 1\right\}\left(\frac{1}{\theta p}-\frac{1}{\mu}\right)\left(\int_{\mathbb{R}^{N}}\left|\Delta u_{n}\right|^{p} d x+\int_{\mathbb{R}^{N}}\left|\nabla u_{n}\right|^{p} d x+\int_{\mathbb{R}^{N}} V(x)\left|u_{n}\right|^{p} d x\right) \\
& -\frac{\lambda \varrho}{\mu} \int_{\mathbb{R}^{N}}\left|u_{n}\right|^{p} d x \\
& \geq \min \left\{d m_{0}, 1\right\}\left(\frac{1}{\theta p}-\frac{1}{\mu}\right)\left\|u_{n}\right\|_{X}^{p}-\frac{\lambda \varrho}{\mu} \int_{\mathbb{R}^{N}}\left|u_{n}\right|^{p} d x \quad \text { for large } n \in \mathbb{N} \text {. }
\end{aligned}
$$

This implies

$$
1 \leq \frac{\lambda \varrho \theta p}{\min \left\{d m_{0}, 1\right\}(\mu-\theta p)} \limsup _{n \rightarrow \infty}\left\|v_{n}\right\|_{L^{p}\left(\mathbb{R}^{N}\right)}^{p}=\frac{\lambda \varrho \theta p}{\min \left\{d m_{0}, 1\right\}(\mu-\theta p)}\|v\|_{L^{p}\left(\mathbb{R}^{N}\right)}^{p} .
$$

Hence, due to (3.15), we see that $v \neq 0$. From the same argument as in Lemma 3.3, we can show that the relations (3.6), (3.7), and (3.8) hold, and hence we conclude that relation (3.9) is true. Therefore we get a contradiction. Thus $\left\{u_{n}\right\}$ is bounded in $X$. This completes the proof.

Next, applying the fountain theorem in [32, Theorem 3.6] with the oddity of $f$, we demonstrate infinitely many weak solutions for problem (P). To do this, let $W$ be a reflexive and separable Banach space. Then there are $\left\{e_{n}\right\} \subseteq W$ and $\left\{f_{n}^{*}\right\} \subseteq W^{*}$ such that

$$
W=\overline{\operatorname{span}\left\{e_{n}: n=1,2, \ldots\right\}}, \quad W^{*}=\overline{\operatorname{span}\left\{f_{n}^{*}: n=1,2, \ldots\right\}},
$$

and

$$
\left\langle f_{i}^{*}, e_{j}\right\rangle= \begin{cases}1 & \text { if } i=j \\ 0 & \text { if } i \neq j\end{cases}
$$

Let us denote $W_{n}=\operatorname{span}\left\{e_{n}\right\}, Y_{k}=\bigoplus_{n=1}^{k} W_{n}$, and $Z_{k}=\overline{\bigoplus_{n=k}^{\infty} W_{n}}$. In order to establish the existence result, we use the following Fountain theorem. 
Lemma 3.6 ([1,32]) Let $X$ be a real reflexive Banach space, $I \in C^{1}(X, \mathbb{R})$ satisfies the $(C)_{c}$ condition for any $c>0$ and I is even. Iffor each sufficiently large $k \in \mathbb{N}$, there exist $\rho_{k}>\delta_{k}>0$ such that the following conditions hold:

(1) $b_{k}:=\inf \left\{I(u): u \in Z_{k},\|u\|_{X}=\delta_{k}\right\} \rightarrow \infty$ as $k \rightarrow \infty$;

(2) $a_{k}:=\max \left\{I(u): u \in Y_{k},\|u\|_{X}=\rho_{k}\right\} \leq 0$.

Then the functional I has an unbounded sequence of critical values, i.e., there exists a sequence $\left\{u_{n}\right\} \subset X$ such that $I^{\prime}\left(u_{n}\right)=0$ and $I\left(u_{n}\right) \rightarrow+\infty$ as $n \rightarrow+\infty$.

Theorem 3.7 Assume that (V), (M1), (M2), (J1)-(J5), and (F1)-(F5) hold. If $\Phi_{0}(x,-v)=$ $\Phi_{0}(x, v)$ holds for all $(x, v) \in \mathbb{R}^{N} \times \mathbb{R}^{N}$ and $f(x,-t)=-f(x, t)$ holds for all $(x, t) \in \mathbb{R}^{N} \times$ $\mathbb{R}$, then for any $\lambda>0$, problem $(\mathrm{P})$ possesses an unbounded sequence of nontrivial weak solutions $\left\{u_{n}\right\}$ in $X$ such that $I_{\lambda}\left(u_{n}\right) \rightarrow \infty$ as $n \rightarrow \infty$.

Proof It is obvious that $I_{\lambda}$ is an even functional and satisfies the $(C)_{c}$ condition. It suffices to show that there exist $\rho_{k}>\delta_{k}>0$ such that

(1) $b_{k}:=\inf \left\{I_{\lambda}(u): u \in Z_{k},\|u\|_{X}=\delta_{k}\right\} \rightarrow \infty \quad$ as $n \rightarrow \infty$;

(2) $a_{k}:=\max \left\{I_{\lambda}(u): u \in Y_{k},\|u\|_{X}=\rho_{k}\right\} \leq 0$,

for $k$ large enough. Denote

$$
\alpha_{k}:=\sup _{u \in Z_{k},\|u\|_{X}=1}\|u\|_{L^{q}\left(\mathbb{R}^{N}\right)}
$$

Then we have $\alpha_{k} \rightarrow 0$ as $k \rightarrow \infty$. In fact, assume to the contrary that there exist $\varepsilon_{0}>0$ and a sequence $\left\{u_{k}\right\}$ in $Z_{k}$ such that

$$
\left\|u_{k}\right\|_{X}=1 \quad \text { and } \quad\left\|u_{k}\right\|_{L^{q}\left(\mathbb{R}^{N}\right)} \geq \varepsilon_{0}
$$

for all $k \geq k_{0}$. By the boundedness of the sequence $\left\{u_{k}\right\}$ in $X$, we can find an element $u \in X$ such that $u_{k} \rightarrow u$ in $X$ as $n \rightarrow \infty$ and

$$
\left\langle f_{j}^{*}, u\right\rangle=\lim _{k \rightarrow \infty}\left\langle f_{j}^{*}, u_{k}\right\rangle=0
$$

for $j=1,2, \ldots$ Thus we deduce $u=0$. However, we see that

$$
\varepsilon_{0} \leq \lim _{k \rightarrow \infty}\left\|u_{k}\right\|_{L^{q\left(\mathbb{R}^{N}\right)}}=\|u\|_{L^{q}\left(\mathbb{R}^{N}\right)}=0,
$$

which is a contradiction.

For any $u \in Z_{k}$, we may suppose that $\|u\|_{X}>1$. According to assumptions (M1), (M2), (J3), and (F2), we obtain that

$$
\begin{aligned}
I_{\lambda}(u)= & \frac{1}{p} \int_{\mathbb{R}^{N}}|\Delta u|^{p} d x+\mathcal{M}\left(\int_{\mathbb{R}^{N}} \Phi_{0}(x, \nabla u) d x\right) \\
& +\frac{1}{p} \int_{\mathbb{R}^{N}} V(x)|u|^{p} d x-\lambda \int_{\mathbb{R}^{N}} F(x, u) d x \\
\geq & \frac{1}{p} \int_{\mathbb{R}^{N}}|\Delta u|^{p} d x+\frac{1}{\theta} M\left(\int_{\mathbb{R}^{N}} \Phi_{0}(x, \nabla u) d x\right) \int_{\mathbb{R}^{N}} \Phi_{0}(x, \nabla u) d x \\
& +\frac{1}{p} \int_{\mathbb{R}^{N}} V(x)|u|^{p} d x-\lambda \int_{\mathbb{R}^{N}}|\rho(x)||u| d x-\lambda \int_{\mathbb{R}^{N}} \frac{|\sigma(x)|}{q}|u|^{q} d x
\end{aligned}
$$




$$
\begin{aligned}
\geq & \frac{\min \left\{1, d m_{0}\right\}}{\theta p}\left(\int_{\mathbb{R}^{N}}|\Delta u|^{p} d x+\int_{\mathbb{R}^{N}}|\nabla u|^{p} d x+\int_{\mathbb{R}^{N}} V(x)|u|^{p} d x\right) \\
& -\lambda \int_{\mathbb{R}^{N}}|\rho(x)||u| d x-\lambda \int_{\mathbb{R}^{N}} \frac{|\sigma(x)|}{q}|u|^{q} d x \\
\geq & \frac{\min \left\{1, d m_{0}\right\}}{\theta p}\|u\|_{X}^{p}-2 \lambda\|\rho\|_{L^{q^{\prime}}\left(\mathbb{R}^{N}\right)}\|u\|_{L^{q}\left(\mathbb{R}^{N}\right)}-\frac{2 \lambda}{q}\|\sigma\|_{L^{\infty}\left(\mathbb{R}^{N}\right)} \int_{\mathbb{R}^{N}}|u|^{q} d x \\
\geq & \frac{\min \left\{1, d m_{0}\right\}}{\theta p}\|u\|_{X}^{p}-2 \lambda C_{4}\|u\|_{X}-\frac{2 \lambda}{q} \alpha_{k}^{q} C_{5}\|u\|_{X}^{q},
\end{aligned}
$$

where $C_{4}$ and $C_{5}$ are positive constants. If we take

$$
\delta_{k}=\left(\frac{2 \lambda C_{5} \alpha_{k}^{q}}{\min \left\{1, d m_{0}\right\}}\right)^{1 /(p-q)},
$$

then $\delta_{k} \rightarrow \infty$ as $k \rightarrow \infty$ because $\theta p<q$ and $\alpha_{k} \rightarrow 0$ as $k \rightarrow \infty$. Hence, if $u \in Z_{k}$ and $\|u\|_{X}=\delta_{k}$, then we conclude that

$$
I_{\lambda}(u) \geq \min \left\{1, d m_{0}\right\}\left(\frac{1}{\theta p}-\frac{1}{q}\right) \delta_{k}^{p}-2 \lambda C_{4} \delta_{k} \rightarrow \infty \quad \text { as } k \rightarrow \infty .
$$

This implies that condition (1) holds.

The proof of condition (2) proceeds analogously as in the proof of [1, Theorem 1.3]. For the reader's convenience, we give the proof. Assume that condition (2) is not true. Then for some $k$ there exists a sequence $\left\{u_{n}\right\}$ in $Y_{k}$ such that

$$
\left\|u_{n}\right\|_{X} \rightarrow \infty \quad \text { as } n \rightarrow \infty \quad \text { and } \quad I_{\lambda}\left(u_{n}\right) \geq 0
$$

Set $w_{n}=u_{n} /\left\|u_{n}\right\|_{X}$. Note that $\left\|w_{n}\right\|_{X}=1$. Since $\operatorname{dim} Y_{k}<\infty$, there exists $w \in Y_{k} \backslash\{0\}$ such that, up to a subsequence,

$$
\left\|w_{n}-w\right\|_{X} \rightarrow 0 \quad \text { and } \quad w_{n}(x) \rightarrow w(x)
$$

for almost all $x \in \mathbb{R}^{N}$ as $n \rightarrow \infty$. If $w(x) \neq 0$, then $\left|u_{n}(x)\right| \rightarrow \infty$ for all $x \in \mathbb{R}^{N}$ as $n \rightarrow \infty$. Hence we obtain by assumption (F4) that

$$
\lim _{n \rightarrow \infty} \frac{F\left(x, u_{n}(x)\right)}{\left\|u_{n}\right\|_{X}^{\theta p}}=\lim _{n \rightarrow \infty} \frac{F\left(x, u_{n}(x)\right)}{\left|u_{n}(x)\right|^{\theta p}}\left|w_{n}(x)\right|^{\theta p}=\infty
$$

for all $x \in \Omega_{2}:=\left\{x \in \mathbb{R}^{N}: w(x) \neq 0\right\}$. As in the proof of Lemma 3.3, we have

$$
\int_{\Omega_{2}} \frac{F\left(x, u_{n}(x)\right)}{\left\|u_{n}\right\|_{X}^{\theta p}} d x \rightarrow \infty \quad \text { as } n \rightarrow \infty
$$

Therefore, we conclude that

$$
\begin{aligned}
I_{\lambda}\left(u_{n}\right) & \leq \frac{1}{p}\left\|u_{n}\right\|_{X}^{p}+\mathcal{M}\left(\int_{\mathbb{R}^{N}} \Phi_{0}\left(x, \nabla u_{n}\right) d x\right)-\lambda \int_{\mathbb{R}^{N}} F\left(x, u_{n}\right) d x \\
& \leq \frac{1}{p}\left\|u_{n}\right\|_{X}^{\theta p}+\mathcal{M}(1)\left(1+\left(\|a\|_{L^{p^{\prime}}\left(\mathbb{R}^{N}\right)}+\frac{b}{p}\right)^{\theta}\right)\left\|u_{n}\right\|_{X}^{\theta p}-\lambda \int_{\mathbb{R}^{N}} F\left(x, u_{n}\right) d x
\end{aligned}
$$




$$
\begin{aligned}
& \leq\left\|u_{n}\right\|_{X}^{\theta p}\left(\frac{1}{p}+\mathcal{M}(1)\left(1+\left(\|a\|_{L^{p^{\prime}}\left(\mathbb{R}^{N}\right)}+\frac{b}{p}\right)^{\theta}\right)-\lambda \int_{\Omega_{2}} \frac{F\left(x, u_{n}(x)\right)}{\left\|u_{n}\right\|_{X}^{p}} d x\right) \\
& \rightarrow-\infty \text { as } n \rightarrow \infty,
\end{aligned}
$$

which contradicts (3.16). This completes the proof.

Remark 3.8 Although we replace (F5) with (F6) in the assumptions of Theorem 3.7, we can show that problem $(\mathrm{P})$ possesses an unbounded sequence of nontrivial weak solutions $\left\{u_{n}\right\}$ in $X$ such that $I_{\lambda}\left(u_{n}\right) \rightarrow \infty$ as $n \rightarrow \infty$.

\section{Appendix: Proof of Lemma 2.5}

In this section, we give a proof of Lemma 2.5 for the reader's convenience. In fact, we consider that it is a well-known fact to researchers in this area.

Proof Let $\left\{u_{n}\right\}$ be a sequence in $X$ such that $u_{n} \rightarrow u$ in $X$ as $n \rightarrow \infty$. Then $\left\{u_{n}\right\}$ is bounded in $X$, and we know the embeddings $X \hookrightarrow L^{p}\left(\mathbb{R}^{N}\right)$ and $X \hookrightarrow L^{q}\left(\mathbb{R}^{N}\right)$ are compact for $p<$ $q<p_{*}$. So we know that

$$
u_{n} \rightarrow u \quad \text { in } L^{p}\left(\mathbb{R}^{N}\right) \quad \text { and } \quad u_{n} \rightarrow u \quad \text { in } L^{q}\left(\mathbb{R}^{N}\right) \text { as } n \rightarrow \infty .
$$

First we prove that $\Psi$ is weakly strongly continuous in $X$. Let $u_{n} \rightarrow u$ in $L^{p}\left(\mathbb{R}^{N}\right) \cap L^{q}\left(\mathbb{R}^{N}\right)$ as $n \rightarrow \infty$. By the convergence principle, there exist a subsequence $\left\{u_{n_{k}}\right\}$ such that $u_{n_{k}}(x) \rightarrow$ $u(x)$ as $k \rightarrow \infty$ for almost all $x \in \mathbb{R}^{N}$ and a function $u_{0} \in L^{p}\left(\mathbb{R}^{N}\right) \cap L^{q}\left(\mathbb{R}^{N}\right)$ such that $\left|u_{n_{k}}(x)\right| \leq u_{0}(x)$ for all $k \in \mathbb{N}$ and for almost all $x \in \mathbb{R}^{N}$. Therefore from (F2), we deduce

$$
\begin{aligned}
\int_{\mathbb{R}^{N}}\left|F\left(x, u_{n_{k}}\right)\right| d x & \leq \int_{\mathbb{R}^{N}} \rho(x)\left|u_{n_{k}}(x)\right|+\frac{\sigma(x)}{q}\left|u_{n_{k}}(x)\right|^{q} d x \\
& \leq\|\rho\|_{L^{q^{\prime}\left(\mathbb{R}^{N}\right)}}\left\|u_{0}\right\|_{L^{q\left(\mathbb{R}^{N}\right)}}+\|\sigma\|_{L^{\infty}\left(\mathbb{R}^{N}\right)}\left\|u_{0}\right\|_{L^{q\left(\mathbb{R}^{N}\right)}}^{q}
\end{aligned}
$$

Since function $f$ satisfies the Carathéodory condition by (F1), we obtain that $F\left(x, u_{n_{k}}\right) \rightarrow$ $F(x, u)$ as $k \rightarrow \infty$ for almost all $x \in \mathbb{R}^{N}$. Therefore, the Lebesgue convergence theorem tells us that

$$
\int_{\mathbb{R}^{N}} F\left(x, u_{n_{k}}\right) d x \rightarrow \int_{\mathbb{R}^{N}} F(x, u) d x
$$

as $k \rightarrow \infty$, which says $\Psi\left(u_{n_{k}}\right) \rightarrow \Psi(u)$ as $k \rightarrow \infty$. Thus $\Psi$ is weakly strongly continuous in $X$.

Next, we show that $\Psi^{\prime}$ is weakly strongly continuous on $X$. By (F2) and Hölder's inequality, we obtain

$$
\begin{aligned}
\int_{\mathbb{R}^{N}}\left|f\left(x, u_{n}\right)-f(x, u)\right|^{q^{\prime}} d x & \leq C_{6} \int_{\mathbb{R}^{N}}\left|f\left(x, u_{n}\right)\right|^{q^{\prime}}+|f(x, u)|^{q^{\prime}} d x \\
& \leq C_{7} \int_{\mathbb{R}^{N}}|\rho(x)|^{q^{\prime}}+\left\||\sigma|^{q^{\prime}}\right\|_{L^{\infty}\left(\mathbb{R}^{N}\right)}\left(\left|u_{n}\right|^{q}+|u|^{q}\right) d x
\end{aligned}
$$

for some positive constants $C_{6}, C_{7}$, which implies that $\left|f\left(x, u_{n}\right)-f(x, u)\right|^{q^{\prime}} \leq g(x)$ for almost all $x \in \mathbb{R}^{N}$ and for some $g \in L^{1}\left(\mathbb{R}^{N}\right)$. Since $u_{n} \rightarrow u$ in $L^{p}\left(\mathbb{R}^{N}\right) \cap L^{q}\left(\mathbb{R}^{N}\right)$ and almost all in 
$\mathbb{R}^{N}$, it follows from (A.1) and the convergence principle that $f\left(x, u_{n}\right) \rightarrow f(x, u)$ for almost all $x \in \mathbb{R}^{N}$. Combining this with the Lebesgue convergence theorem, we have

$$
\begin{aligned}
\left\|\Psi^{\prime}\left(u_{n}\right)-\Psi^{\prime}(u)\right\|_{X^{*}} & =\sup _{\|v\|_{X} \leq 1}\left|\left\langle\Psi^{\prime}\left(u_{n}\right)-\Psi^{\prime}(u), v\right\rangle\right| \\
& =\sup _{\|v\|_{X} \leq 1} \int_{\mathbb{R}^{N}}\left|f\left(x, u_{n}\right)-f(x, u)\right||v| d x \\
& \leq\left(\int_{\mathbb{R}^{N}}\left|f\left(x, u_{n}\right)-f(x, u)\right|^{q^{\prime}} d x\right)^{\frac{1}{q^{\prime}}} \rightarrow 0 \quad \text { as } n \rightarrow \infty .
\end{aligned}
$$

Therefore, we derive that $\Psi^{\prime}\left(u_{n}\right) \rightarrow \Psi^{\prime}(u)$ in $X$. This completes the proof.

\section{Acknowledgements}

The authors wish to express their sincere thanks to the anonymous referees and the handling editor for many constructive comments, leading to the improved version of this paper.

\section{Funding}

The first author was supported by Basic Science Research Program through the National Research Foundation of Korea funded by the Ministry of Education (NRF-2017R1D1A1B03031104). J.-M. Kim was supported by National Research Foundation of Korea Grant funded by the Korean Government (NRF-2016R1D1A1B03930422). J. Lee was supported by the Basic Science Research Program through the National Research Foundation of Korea (NRF) funded by the Ministry of Education (2018R1D1A1B07048620) and (2019R1A6A1A1 1051177). K. Park was supported by Basic Science Research Program through the National Research Foundation of Korea (NRF) funded by the Ministry of Science, ICT and Future Planning (2017R1E1A1A03070225).

\section{Availability of data and materials}

Not applicable.

\section{Competing interests}

The authors declare that they have no competing interests.

\section{Authors' contributions}

All authors contributed equally to the writing of this paper. All authors read and approved the final manuscript.

\section{Author details}

'Department of Mathematics, Sungkyunkwan University, Suwon, Republic of Korea. ${ }^{2}$ Department of Mathematics, Yonsei University, Seoul, Republic of Korea. ${ }^{3}$ Institute of Mathematical Sciences, Ewha Womans University, Seoul, Republic of Korea. ${ }^{4}$ Department of Mathematics, Incheon National University, Incheon, Republic of Korea.

\section{Publisher's Note}

Springer Nature remains neutral with regard to jurisdictional claims in published maps and institutional affiliations.

Received: 21 March 2019 Accepted: 30 June 2019 Published online: 12 July 2019

\section{References}

1. Alves, C.O., Liu, S.B.: On superlinear p(x)-Laplacian equations in $\mathbb{R}^{N}$. Nonlinear Anal. 73, 2566-2579 (2010)

2. Ambrosetti, A., Rabinowitz, P.: Dual variational methods in critical point theory and applications. J. Funct. Anal. 14 349-381 (1973)

3. Arosio, A.: A geometrical nonlinear correction to the Timoshenko beam equation. Nonlinear Anal. 47, 729-740 (2001)

4. Ball, J.M.: Initial boundary value problem for an extensible beam. J. Math. Anal. Appl. 42,61-90 (1973)

5. Ball, J.M.: Stability theory for an extensible beam. J. Differ. Equ. 14, 399-418 (1973)

6. Barletta, G., Livrea, R.: Infinitely many solutions for a class of differential inclusions involving the $p$-biharmonic. Differ. Integral Equ. 26, 1157-1167 (2013)

7. Berger, H.M.: A new approach to the analysis of large deflections of plates. J. Appl. Mech. 22, 465-472 (1955)

8. Bernis, F., Azorero, J.G., Peral, I.: Existence and multiplicity of nontrivial solutions in semilinear critical problems of fourth order. Adv. Differ. Equ. 1, 219-240 (1996)

9. Bonanno, G., Di Bella, B.: A boundary value problem for fourth-order elastic beam equations. J. Math. Anal. Appl. 343, $1166-1176(2008)$

10. Boureanu, M.-M., Preda, F.: Infinitely many solutions for elliptic problems with variable exponent and nonlinear boundary conditions. Nonlinear Differ. Equ. Appl. 19, 235-251 (2012)

11. Cammaroto, F., Vilasi, L.: Multiple solutions for a Kirchhoff-type problem involving the $p(x)$-Laplacian operator. Nonlinear Anal. 74, 1841-1852 (2011)

12. Candito, P., Li, L., Livrea, R.: Infinitely many solutions for a perturbed nonlinear Navier boundary value problem involving the $p$-biharmonic. Nonlinear Anal. 75, 6360-6369 (2012) 
13. Chabrowski, J., do Ó, J.M.: On some fourth-order semilinear elliptic problems in $\mathbb{R}^{N}$. Nonlinear Anal. 49, 861-884 (2002)

14. De Napoli, P., Mariani, M.: Mountain pass solutions to equations of $p$-Laplacian type. Nonlinear Anal. 54, 1205-1219 (2003)

15. Drabek, P., Otani, M.: Global bifurcation result for the $p$-biharmonic operator. Electron. J. Differ. Equ. 2001, 48, 1-19 (2001)

16. Dreher, M.: The Kirchhoff equation for the $p$-Laplacian. Rend. Semin. Mat. (Torino) 64, 217-238 (2006)

17. Fan, X., Han, X.: Existence and multiplicity of solutions for $p(x)$-Laplacian equations in $\mathbb{R}^{N}$. Nonlinear Anal. 59, 173-188 (2004)

18. Guo, Y., Nie, J.: Existence and multiplicity of nontrivial solutions for $p$-Laplacian Schrödinger-Kirchhoff-type equations J. Math. Anal. Appl. 428, 1054-1069 (2015)

19. Kim, Y.-H., Bae, J.-H., Lee, J.: The existence of infinitely many solutions for nonlinear elliptic equations involving p-Laplace type operators in $\mathbb{R}^{N}$. J. Nonlinear Sci. Appl. 10, 2144-2161 (2017)

20. Kirchhoff, G.: Mechanik. Teubner, Leipzig (1883)

21. Li, C., Tang, C.-L.: Three solutions for a Navier boundary value problem involving the $p$-biharmonic. Nonlinear Anal. 72 , 1339-1347 (2010)

22. Li, L., Ding, L., Pan, W.-W.: Existence of multiple solutions for a $p(x)$-biharmonic equation. Electron. J. Differ. Equ., 2013, $139(2013)$

23. Lin, X., Tang, X.H.: Existence of infinitely many solutions for $p$-Laplacian equations in $\mathbb{R}^{N}$. Nonlinear Anal. 92, 72-81 (2013)

24. Liu, L., Chen, C.: Infinitely many solutions for $p$-biharmonic equation with general potential and concave-convex nonlinearity in $\mathbb{R}^{N}$. Bound. Value Probl. 2016, $61-9$ (2016)

25. Liu, S.B.: On ground states of superlinear $p$-Laplacian equations in $\mathbb{R}^{N}$. J. Math. Anal. Appl. 361, 48-58 (2010)

26. Liu, S.B., Li, S.J.: Infinitely many solutions for a superlinear elliptic equation. Acta Math. Sinica (Chin. Ser.) 46, 625-630 (2003)

27. Micheletti, A.M., Pistoia, A.: Multiplicity results for a fourth-order semilinear elliptic problem. Nonlinear Anal. 31, 895-908 (1998)

28. Mihăilescu, M., Rădulescu, V.: A multiplicity result for a nonlinear degenerate problem arising in the theory of electrorheological fluids. Proc. R. Soc., Math. Phys. Eng. Sci. 462, 2625-2641 (2006)

29. Miyagaki, O.H., Souto, M.A.S.: Superlinear problems without Ambrosetti and Rabinowitz growth condition. J. Differ. Equ. 245, 3628-3638 (2008)

30. Sun, J., Chu, J., Wu, T.-F.: Existence and multiplicity of nontrivial solutions for some biharmonic equations with p-Laplacian. J. Differ. Equ. 262, 945-977 (2017)

31. Talbi, M., Tsouli, N.: On the spectrum of the weighted $p$-biharmonic operator with weight. Mediterr. J. Math. 4, 73-86 (2007)

32. Willem, M.: Minimax Theorems. Progress in Nonlinear Differential Equations and Their Applications, vol. 24, p. x+162 pp. Birkhäuser, Boston (1996)

33. Woinowsky-Krieger, S.: The effect of axial force on the vibration of hinged bars. J. Appl. Mech. 17, 35-36 (1950)

34. Xiu, Z., Zhao, J., Chen, J., Li, S.: Multiple solutions on a p-biharmonic equation with nonlocal term. Bound. Value Probl. $2016,154,1-11(2016)$

\section{Submit your manuscript to a SpringerOpen ${ }^{\circ}$ journal and benefit from:}

- Convenient online submission

- Rigorous peer review

- Open access: articles freely available online

- High visibility within the field

- Retaining the copyright to your article

Submit your next manuscript at $>$ springeropen.com 